\title{
A THERMO-PNEUMATIC ACTUATION PRINCIPLE FOR A MICROMINIATURE PUMP AND OTHER MICROMECHANICAL DEVICES
}

F. C. M. VAN DE POL, D. G. J. WONNINK, M. ELWENSPOEK and J. H. J. FLUITMAN

Department of Electrical Engineering, University of Twente, P.O. Box 217 , 7500 AE Enschede (The Netherlands)

\section{Abstract}

A new type of actuator for a microminiature pump is presented. The pressure of air in a cavity, raised by resistive heating, deflects a thin silicon membrane. The dynamics of membrane deflection is studied experimentally, the results being in excellent agreement with simulation. We conclude that the device is suitable as an actuator in a micro-miniature pump.

Introduction

Since 1983, research on microminiature pumps has been carried out at the University of Twente. Several prototypes were realized in silicon, all piezoelectrically driven [1]. Major drawbacks of piezoelectric actuation are the necessity for high electrical voltages (100 V or more) and the unavailability of piezoelectric thin-film materials suitable for operation at low frequencies $(10 \mathrm{~Hz}$ or less). Considering various actuation principles [2], it was concluded that thermo-pneumatic actuation, as we call it, might be a good alternative.

In general, we define a thermo-pneumatic actuator as a device consisting of a sealed cavity filled with a thermally expandable medium that can be heated or cooled down, resulting in a pressure change in the cavity. This induced pressure change is used to actuate a flexible or movable part, like a membrane or piston. Gas or a gas/liquid system can be used as the expanding medium. A temperature change in the cavity can be achieved by resistive heating, the use of a Peltier element or a laser. This actuation principle has been used in a microminiature valve, using a gas/liquid system and resistive heating [3]. This paper reports on the realization, behaviour and simulation of a thermo-pneumatic actuator designed to drive a microminiature pump.

Figure 1 shows a sketch of the actuator, comprising a cavity filled with air and a built-in aluminium meander that serves as a heater resistor. The resistor is supported by a thin silicon sheet suspended by four small silicon beams, which serve as a restriction to the heat flow. Aluminium current leads 

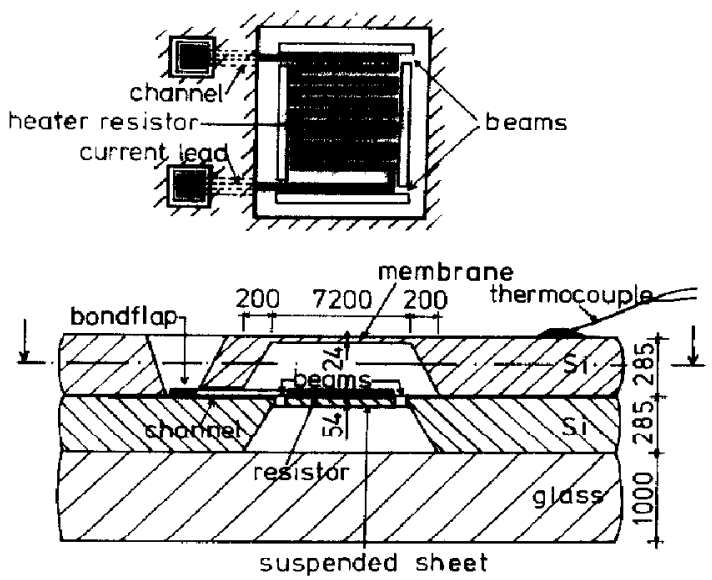

Fig. 1. Sketch of the thermo-pneumatic actuator (dimensions in $\mu \mathrm{m}$ ).

connecting the meander to the bond flaps run through narrow channels, which form a restriction to the gas flow. This actuator is intended to be mounted on a pump, such that the flexible membrane can displace liquid present in a pump chamber.

\section{Simulation model}

Bond graph techniques [4] and TUTSIM [5] are used for physical modelling and simulation of the dynamic behaviour of the actuator. The model comprises the thermal behaviour of the actuator, the thermodynamics of the gas, the fluid dynamics of the channels and the mechanics of the flexible membrane. A detailed description of the simulation model will be reported elsewhere [2].

\section{Experiments}

Starting materials for the actuator are two silicon (100) 2 inch wafers, polished on both sides, and a Duran borosilicate glass wafer. The silicon wafers are shaped by wet chemical etching in a $\mathrm{KOH}$ water solution using standard photolithography for pattern definition. The glass wafer is cut out off a plate and polished (surface roughness $<0.05 \mu \mathrm{m}$ ). The wafers are attached to one another by means of anodic bonding $[6,7]$. To achieve a bond between the two silicon wafers, an intermediate layer of silicon oxide and sputtered borosilicate glass is required. A thermocouple is attached to the upper silicon wafer to monitor the temperature of the actuator as a whole.

Deflection of the flexible membrane as a function of power dissipated in the resistor is measured dynamically using a DEKTAK 3030 surface profiler scanning over $50 \mu \mathrm{m}$ during $50 \mathrm{~s}$ with the scanning needle approx- 
imately at the centre of the membrane. The scan length being small compared with the membrane width, the needle remains virtually in the same position. Thus, deflection at the centre of the membrane, $w_{0}$, versus time is plotted directly.

\section{Results and discussion}

Figure 2 shows $w_{0}$ as a function of time, $t$, for a number of d.c. voltages, $U$, applied to the resistor. The voltage is switched on at time $t=4 \mathrm{~s}$. A fast increase with a relaxation time $\tau_{t}$ is followed by a slower decrease to zero deflection, with a relaxation time $\tau_{\mathrm{p}}$. The first fast increase is caused by a temperature rise in the cavity and a related pressure increase. The relaxation time for warming up the cavity is mainly determined by the thermal conductance, $G_{\mathrm{ta}}$, of the air in the cavity, the thermal conductance, $G_{\mathrm{tb}}$, of the small suspending beams, and by the total heat capacity of the cavity. Since the heat capacity of the air in the cavity is negligible, this total heat capacity approximately equals the heat capacity, $C_{t}$, of the suspended thin silicon sheet supporting the resistor. Maximum temperature and pressure build-up and related deflection $w_{0}$ are determined by input power and thermal conductances $G_{\mathrm{ta}}$ and $G_{\mathrm{tb}}$.

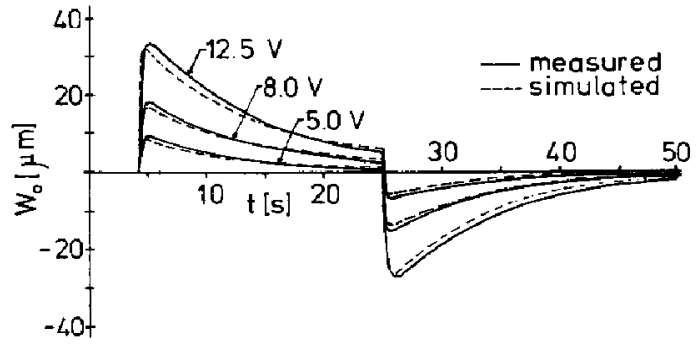

Fig. 2. Deflection $w_{0} v s$. time for a number of applied voltages.

The subsequent slower decrease of $w_{0}$ is due to a flow of gas out of the cavity and a related pressure decrease. $\tau_{p}$ should be determined by the volume of the cavity, $V_{\mathrm{p}}$, and the flow conductances, $G_{\mathrm{pc}}$, of the channels. However, the calculated value of $G_{p c}$ is much too low to account for $\tau_{p}$. Evidently there is some leak in our test device, with a flow conductance $G_{\mathrm{pl}}$, which was confirmed by the observation of a partial failure during the anodic bonding of the two silicon wafers.

When the d.c. voltage is switched off at time $t=25 \mathrm{~s}$, the opposite behaviour is observed. The cavity cools down, causing a pressure drop in the cavity and a related fast decrease of $w_{0}$, with a relaxation time $\tau_{t}{ }^{\prime}$ approximately equalling $\tau_{t}$. The following slower increase to zero deflection is due to a flow of gas into the cavity, with a relaxation time $\tau_{\mathrm{p}}$ ' determined by $V_{\mathrm{p}}$ and $G_{\mathrm{pl}}$, and therefore approximately equalling $\tau_{\mathrm{p}}$. The dashed lines in Fig. 2 represent results of simulations. Table 1 summarizes some parameter values. 
TABLE 1

Calculated and measured parameter values

\begin{tabular}{|c|c|c|c|c|c|}
\hline Parameter & & $\begin{array}{l}\text { Measured } \\
\text { (plots) }\end{array}$ & $\begin{array}{l}\text { Calculated } \\
\text { (geometry) }\end{array}$ & Simulated & Units \\
\hline $\begin{array}{l}\text { Volume cavity } \\
\text { Flow conduct. channels } \\
\text { Flow conduct. leak } \\
\text { Relaxation time } \\
\text { Relaxation time } \\
\text { Relaxation time }\end{array}$ & $\begin{array}{l}V_{\mathrm{p}} \\
G_{\mathrm{pc}} \\
G_{\mathrm{pl}} \\
\tau_{\mathbf{p}} \\
\tau_{\mathbf{p}} \\
\tau_{\mathbf{p}}^{\prime \prime}\end{array}$ & $\begin{array}{r}10 \\
11 \\
9\end{array}$ & $\begin{array}{l}2.7 \times 10^{-8} \\
4.0 \times 10^{-16}\end{array}$ & $\begin{array}{l}2.7 \times 10^{-8} \\
8.0 \times 10^{-14} \\
9 \\
9 \\
9\end{array}$ & $\begin{array}{l}\mathrm{m}^{3} \\
\mathrm{~m}^{5} / \mathrm{N} \mathrm{s} \\
\mathrm{m}^{5} / \mathrm{N} \mathrm{s} \\
\mathrm{s} \\
\mathrm{s} \\
\mathrm{s}\end{array}$ \\
\hline $\begin{array}{l}\text { Heat capacity sheet } \\
\text { Heat conductivity, air } \\
\text { Heat conductivity, beams } \\
\text { Response time } \\
\text { Response time }\end{array}$ & $\begin{array}{l}C_{t} \\
G_{\text {ta }} \\
G_{\text {tb }} \\
\tau_{t} \\
\tau_{t}^{\prime}\end{array}$ & $\begin{array}{l}0.25 \\
0.25\end{array}$ & $\begin{array}{l}4.4 \times 10^{-3} \\
1.2 \times 10^{-2} \\
1.5 \times 10^{-2} \\
0.16 \\
0.16\end{array}$ & $\begin{array}{l}6.0 \times 10^{-3} \\
1.2 \times 10^{-2} \\
1.8 \times 10^{-2} \\
0.20 \\
0.20\end{array}$ & $\begin{array}{l}\mathbf{J} / \mathbf{K} \\
\mathbf{W} / \mathbf{K} \\
\mathbf{W} / \mathbf{K} \\
\mathbf{s} \\
\mathbf{s}\end{array}$ \\
\hline
\end{tabular}

Figure 3 shows $w_{0}$ when a block voltage of $12.9 \mathrm{~V}$ is applied to the resistor at $0.1 \mathrm{~Hz}$. The system moves towards a steady state with a relaxation time $\tau_{p}{ }^{\prime \prime}$, which is also determined by the volume of the cavity, $V_{p}$, and the flow conductance of the leak, $G_{\mathrm{pl}}$; therefore $\tau_{\mathrm{p}}$ " approximately equals $\tau_{\mathrm{p}}$ ' and $\tau_{\mathrm{p}}$. After $30 \mathrm{~s}$ this steady state is reached with an amplitude $A_{\mathrm{m}}$ of 23 $\mu \mathrm{m}$. The dashed line corresponds to a simulation.

At higher frequencies the actuator shows a similar behaviour. However, at frequencies exceeding $0.4 \mathrm{~Hz}$, there is no time to reach maximum or minimum $w_{0}$, resulting in a decrease of $A_{\mathrm{m}}$. Therefore, the maximum of $A_{\mathrm{m}}$ is determined by input power, $G_{\mathrm{ta}}$ and $G_{\mathrm{tb}}$, and frequency. In Fig. 4 measured and simulated amplitudes are given as a function of frequency, for a block voltage of $12.9 \mathrm{~V}$.

The temperature rise of the actuator as a whole, as determined by means of the attached thermocouple, appears to be slow and small. For instance, a maximum temperature rise of $10 \mathrm{~K}$ is reached after $5 \mathrm{~min}$ at an input power of $0.5 \mathrm{~W}$.

In order to determine the influence of the presence of a liquid on the behaviour of the actuator, to check its suitability for application in a pump,
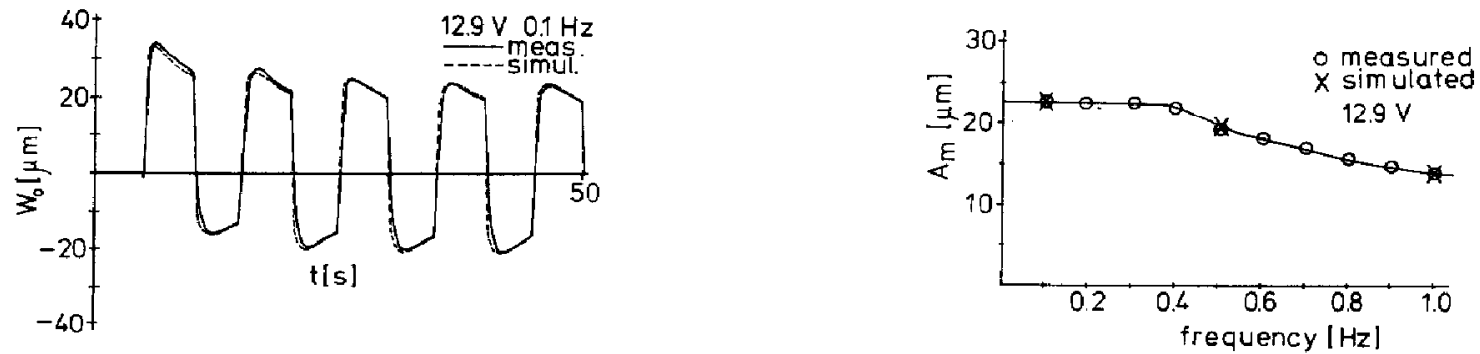

Fig. 3. Deflection $w_{0}$ vs. time for a block voltage of $12.9 \mathrm{~V}$ and $0.1 \mathrm{~Hz}$.

Fig. 4. Measured and simulated amplitudes $A_{\mathrm{m}}$ vs. frequency for a block voltage of $12.9 \mathrm{~V}$. 
the membrane was covered with a drop of water. The presence of this water has no measurable effect on $w_{0}$ or $\boldsymbol{A}_{\mathrm{m}}$.

A deflection of $23 \mu \mathrm{m}$ corresponds to a pressure difference over the membrane of $0.02 \mathrm{~atm}$ and a volume stroke of $0.5 \mu \mathrm{l}$. Reduction of the thermal conductances $G_{\text {ta }}$ and $G_{\text {tb }}$ will result in an increase in temperature and pressure build-up.

If the actuator is to operate at frequencies exceeding $0.4 \mathrm{~Hz}$, the relaxation times $\tau$ and $\tau^{\prime}$ have to be reduced. This can be achieved by reducing the heat capacity $C_{\mathrm{t}}$ of the suspended thin silicon sheet.

\section{Conclusion}

The actuator behaves as expected: results of simulations and measurements coincide within $10 \%$. Hence the simulation model can be used for optimizing the actuator for a required pressure build-up in the cavity and a specific related (free) volume stroke of the membrane.

Important parameters are the response time $\tau_{t}$ (determining maximum frequency $f_{\max }$ ) and the maximum build-up pressure $\Delta p_{\max }$. The heat capacity of the suspended thin silicon sheet supporting the aluminium resistor determines, to a great extent, $\tau_{t}$ and $f_{\max }$, while $\Delta p_{\max }$ is mainly determined by input power and thermal conductances. Both $\Delta p_{\max }$ and the induced membrane deflection are sufficient to drive the microminiature pump we have in mind, and can be even further optimized.

The construction of the actuator is simple and it can be fabricated using IC-compatible materials.

\section{Acknowledgements}

The authors would like to thank Peter Breedveld, Rineke Groothengel, Joep Molkenboer and John Baxter for their help and contributions.

\section{References}

1 H. T. G. van Lintel, F. C. M. van de Pol and S. Bouwstra, A piezoelectric micropump based on micromachining of silicon, Sensors and Actuators, 15 (1988) 153 - 167.

2 F. C. M. van de Pol et al, to be published.

3 M. J. Zdeblick and J. B. Angell, A microminiature electric-to-fluidic valve, Proc. 4th Int. Conf. on Solid-State Sensors and Actuators, Transducers '87, Tokyo, Japan, June $2 \cdot 5,1987$, pp. $827-829$.

4 A. M. Bos and P. C. Breedveld, 1985 Update of the bond graph bibliography, $J$. Franklin Inst., 319 (1985) 269 - 286.

5 TUTSIM, a commercially available simulation program, developed at the University of Twente.

6 G. Wallis and D. I. Pomerantz, Field assisted glass-metal sealing, J. Appl. Phys., 40 (1969) 3946 - 3949.

7 A. Hanneborg and P. A. Ohlckers, Anodic bonding of silicon chips using sputterdeposited Pyrex 7740 thin films, Proc. 12th Nordic Semiconductor Meeting, Jeunaker, Norway, June 8-11, 1986, pp. $290-293$. 